\title{
Reduction of $\mathrm{PtO}_{2}$ Powders (Adam's Catalyst) under Electron Beam Irradiation
}

Jingyue Liu

Science \&Technology, Monsanto Company, 800 N. Lindbergh Blvd., St. Louis, MO 63167

Under certain conditions, noble metals can form relatively stable oxides. For platinum, for example, the oxide phases include $\mathrm{PtO}, \square-\mathrm{PtO}_{2}, \square-\mathrm{PtO}_{2}, \mathrm{Pt}_{3} \mathrm{O}_{4}$, and $\mathrm{Pt}_{3} \mathrm{O}_{8}$; these platinum oxide phases possess unique catalytic, electrical, or optical properties. The platinum dioxide powders, also called the Adam's catalyst, have been used extensively as a catalyst for hydrogenation reactions. The Adam's catalyst consists mostly of crystalline $\square-\mathrm{PtO}_{2}$ phase. Platinum dioxide decomposes upon heating into pure $\mathrm{Pt}$ at temperatures $>500^{\circ} \mathrm{C}$ and can be easily reduced by carbon monoxide, hydrogen, or other reducing agents. The Adam's catalyst has a surface area of about $80 \mathrm{~m}^{2} / \mathrm{gm}$; it usually consists of flakes, platelets, powders, and nano-ribbons. In addition to be an important hydrogenation catalyst, platinum oxide films also find applications in microelectronics, chemical sensors, optical switches, and passivation of high-Tc superconductors. Surprisingly, however, there is not much study on the nanostructure of Adam's catalyst or platinum oxide films.

During our study of the nanostructure of $\mathrm{PtO}_{2}$ powders, we found that $\mathrm{PtO}_{2}$ crystals can be easily reduced under electron beam irradiation. We investigated this reduction process of noble metal oxides with the goal of understanding the oxygen evolution and the formation of Pt nanoparticles during the electron-beam-induced reduction processes. We used electron energy loss spectroscopy (EELS), high-angle annular dark-field (HAADF) imaging, and atomic resolution TEM techniques to examine the structural and chemical changes of $\mathrm{PtO}_{2}$ nanocrystals during the reduction process. A field emission STEM/TEM (200 kV) equipped with a post column GIF was used for obtaining ELLS spectra and images. The HAADF images and EELS spectra were acquired with a probe size of $0.2 \mathrm{~nm}$ and a beam current of about $15 \mathrm{pA}$.

Figure 1 shows a HAADF image of a $\mathrm{PtO}_{2}$ crystal after about 2 minutes of electron beam irradiation, clearly revealing the structural modification of the original $\mathrm{PtO}_{2}$ flake. The bright nanoparticles may represent metallic $\mathrm{Pt}$ and the gray areas may represent amorphous or highly disordered platinum oxide phases. The degree and rate of reduction depend on the morphology of the $\mathrm{PtO}_{2}$ nanocrystals, the sample thickness, and the electron probe parameters. For example, we found that it is difficult to completely reduce the $\mathrm{PtO}_{2}$ nano-ribbons present in the Adam's catalyst, which may contain more than one phases of platinum oxides. To monitor the oxygen evolution during the reduction process, figure 2 shows a time series of EELS spectra recorded after various periods of electron beam

irradiation. Due to the electron irradiation, the oxygen K-edge changes in shape and shifts to higher energies, which is an indication of electron-induced reduction; the change in the peak intensity of the oxygen K-edge represents the loss of oxygen of the irradiated area. The fine structure of the oxygen $\mathrm{K}$-edge provides the fingerprint of the bonding properties of Pt. After about 30 minutes, the $\mathrm{PtO}_{2}$ was completely reduced to metallic $\operatorname{Pt}(\operatorname{spectrum} f)$ and small Pt nanoparticles were observed.

Figure 3 is an atomic resolution TEM image of a $\mathrm{PtO}_{2}$ crystal after prolonged electron beam irradiation, clearly showing the amorphous, highly disordered, and metallic Pt phases. Point defects, twin boundaries, and other types of defects can form within the growing Pt nanoparticles. With an intense electron beam of nanometer size, we may be able to directly fabricate electrically conducting Pt nano-wires into insulating $\mathrm{PtO}_{2}$ films; the electron beam patterning of noble metal-oxide films 
might become a useful method for metallization and fabrication of electrical interconnects in nanoelectronics and nanotechnology. The reduction processes of $\mathrm{PtO}_{2}$ and the formation and structural evolution of Pt nanoparticles will be discussed.
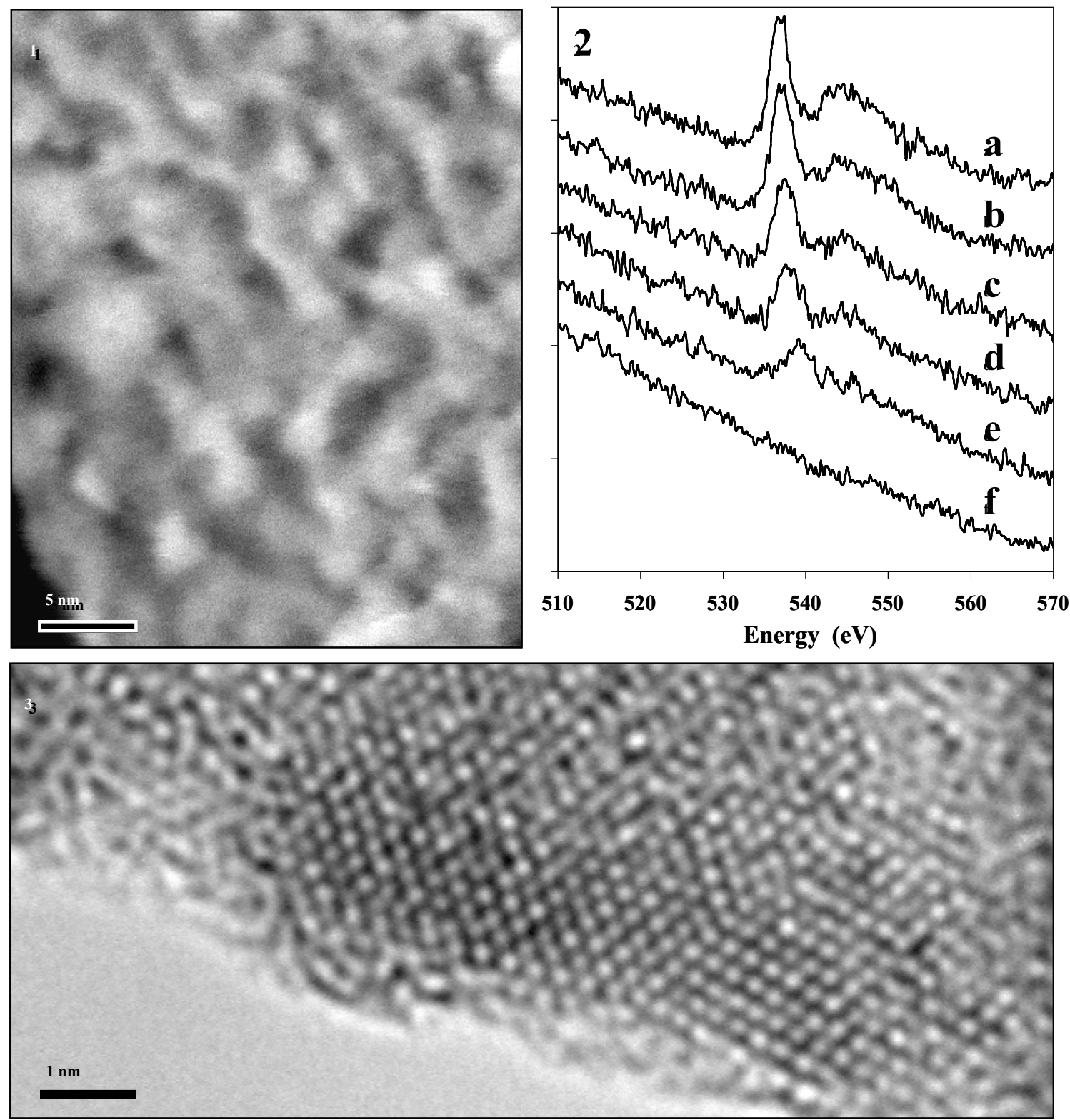

Fig. 1 High-resolution HAADF image of a $\mathrm{PtO}_{2}$ crystal after about 2 minutes of electron beam irradiation shows the formation of small Pt nanoparticles.

Fig. 2 PEELS spectra of $\mathrm{PtO}_{2}$ recorded after various periods of electron beam irradiation show the progressive reduction of the $\mathrm{PtO}_{2}$ crystal. Pure $\mathrm{Pt}$ metal was obtained after about 30 minutes of electron beam irradiation (scanning mode) as shown in the spectrum $\mathrm{f}$.

Fig. 3 Atomic resolution TEM image of $\mathrm{PtO}_{2}$ recorded after prolonged electron irradiation shows the formation of nanocrystalline Pt and the amorphous or highly disordered intermediate oxide phases. Also note the point defects and the twin boundary in the Pt nanoparticle. 